\title{
Correspondence
}

\section{The unanticipated difficult airway}

To the Editor:

The article by Crosby $e t a l$ "The unanticipated difficult airway with recommendations for management"l and accompanying editorial "The difficult airway - a Canadian perspective" are important contributions. Four important points deserve additional commentary.

First, both articles considered and discussed the most useful and accurate definition of a difficult intubation and both politely indicated that the ASA definition of "more than three attempts" or "more than ten minutes" is lacking. I agree: a difficult intubation can be both time and number of attempts independent. ${ }^{3}$ The definition by Crosby of "I) more than two attempts with the same blade or; 2) a change in the blade or an adjunct to a direct laryngoscopy or; 3 ) use of an alternative device or technique following failed intubation with direct laryngoscopy" comes close to covering the clinical realities of "difficulty" for everyone. I would add "a Grade IIIB or Grade IV view". Grade III should be divided up into Grade IIIA = only proximal and distal half of epiglottis seen and Grade IIIB = only proximal half (tip) of epiglottis seen, because these views probably respond differently to therapeutic manoeuvres such as optimal external laryngeal manipulation and change in length and type of blade. ${ }^{3}$

Second, Finucane characterized an algorithm for the management of the unexpected difficult airway in the Crosby et al. article as a "simplified version of that presented in the ASA guideline." I want to emphasize that "simpler" should not mean "different" because intellectually and clinically both algorithms are the same.

Third, Finucane and Crosby emphasize the importance of teaching airway management skills and I am as puzzled as they by the fact that the majority of teaching programs in North America do not have a structured teaching program in this area. The only explanation I have for such an illogical situation is that most older anesthesiologists come from an era when they and others thought that all anesthesiologists were the 'airway experts', so why would 'airway experts' need more or different training? The ASA Closed Claim. Study showed anesthesiologists were not 'expert' enough to prevent complications due to management of the airway from being the most frequent and severe of all complications related to the administration of anesthesia. Our program at UCSD in patients has worked well but the supervising faculty must exercise tight control, good common sense and judgement. ${ }^{4}$ We use mannequins only in the beginning of a formal airway rotation to help the resident get off to a better start in patients. Thus, I do not feel informed consent is necessary because the anesthesia and airway care that is administered is as good as or better, from both trauma and success points of view, as they might otherwise receive. ${ }^{5}$

Finally, I am puzzled by Dr. Finucane's characterization of Crosby's article as "displaying a distinctively Canadian viewpoint". I do not argue that such a thing exists but what is it? I was very relieved to learn that important local / territorial differences do not exist because it is hard to imagine that such a fundamentally important life-taking/life-giving problem as the management of the difficult airway would have fundamentally different answers.

Jonathan L. Benumof MD

San Diego, California, USA

\section{REFERENCES}

1 Crosby ET, Cooper RM, Douglas MJ, et al. The unanticipated difficult airway with recommendations for management. Can J Anaesth 1998; 45: 757-76.

2 Finucane B. The difficult airway - a Canadian perspective (Editorial). Can J Anaesth 1998; 45: 713-8.

3 Benumof $J$. Difficult laryngoscopy: obtaining the best view (Editorial). Can J Anaesth 1994; 41: 361-5.

4 Cooper SD, Benumof $J$. Teaching management of the airway: The UCSD airway rotation. In: Benumof JL (Ed.). Airway Management: Principles and Practice. Philadelphia, PA: Mosby CV, 1996: 903-10.

5 Benumof $J L$, Cooper $S D$. Teaching airway management skills: what about patient consent? Anaesthesia 1996; 85: 438-9.

\section{REPLY:}

I would like to thank Dr. Benumof for his interest and thoughtful commentary. I think that we should teach what we practice. We rely, in many instances, on technology and techniques that we know are dated and less than optimum for the task at hand. Rose reported that difficult laryngoscopy was most often managed with persistent attempts at direct laryngoscopy and that the use of alternative approaches to tracheal intubation was uncommon. I In a recent survey, Rosenblatt presented 